\title{
Extensible Flexible Optical System for Nano-scale Remote Sensing Satellite “PRISM”
}

\author{
By Yuki SATO, Sang Kyun KIM, Yasuhiro KUSAKAWA, Kensuke SHIMIZU, Takashi TANAKA, \\ Mitsuhito KOMATSU, Casey LAMBERT and Shinichi NAKASUKA
}

Department of Aeronautics and Astronautics, The University of Tokyo, Japan

(Received May 2nd, 2008)

\begin{abstract}
As traditional satellites successfully utilize extensible structures such as long magnetic booms or large antennae, the variety of space missions to be realized by nano-scale satellites would be greatly expanded if they employed such structures. Specifically for remote sensing applications, extensible structures are convenient for achieving long focal lengths required by the optics system. In our laboratory, a nano-scale remote sensing satellite "PRISM" has been developed with an expected launch date in the 2008 fiscal year. PRISM has an extensible boom with a telephoto lens at its tip, creating a refractive telescope, which on the orbit can acquire earth images with a resolution better than $30 \mathrm{~m}$. In this paper, we describe the design of the boom and present results from a micro-gravity experiment where the operation and system identification tests were performed.
\end{abstract}

Key Words: Extensible Space Structure, Flexible Optical System, PRISM

\section{Introduction}

Since the beginning of space technology development, various kinds of deployable space structures have been studied; however, their application to nano-scale satellites has not been achieved. As traditional satellites with a deployable structure have been successful in the past, it is thought that very small satellites could significantly extend their mission capabilities by utilizing such structures. With respect to space optics, the extension of structural elements enables the long focal lengths required to take high resolution images. In the past, several satellites have achieved successful deployment of extensible optical systems on orbit ${ }^{1)}$; however this has yet to be achieved with nano-satellite such as our previous satellites XI-IV ${ }^{2)}$ or $\mathrm{XI}-\mathrm{V}^{3)}$.

At the Intelligent Space Systems Laboratory (ISSL) of the University of Tokyo, a nano-scale remote sensing satellite named PRISM has been developed by students. The satellite is designed to acquire earth images with a resolution better than $30 \mathrm{~m}$ by extending a telescopic lens structure on a light body, about $8 \mathrm{~kg}$. The telescope is stowed during its launch into space, and once it becomes extended in-orbit, it will have a much longer focal length than other nano-scale satellites.

The PRISM project started in 2001 and the extensible boom has been comprehensively studied since that time. Following the satellite's breadboard model design stage, the design for base of the boom structure was fixed. The next step was to complete the design for the telescope satisfying the mission's optical specifications.

In this paper, we will initially present the technological challenges that were solved during the development of engineering model. Secondly, the results of testing on a micro-gravity flight will be described. Finally, a discussion of our future work related to the launch of PRISM, and our design methodology will be presented.

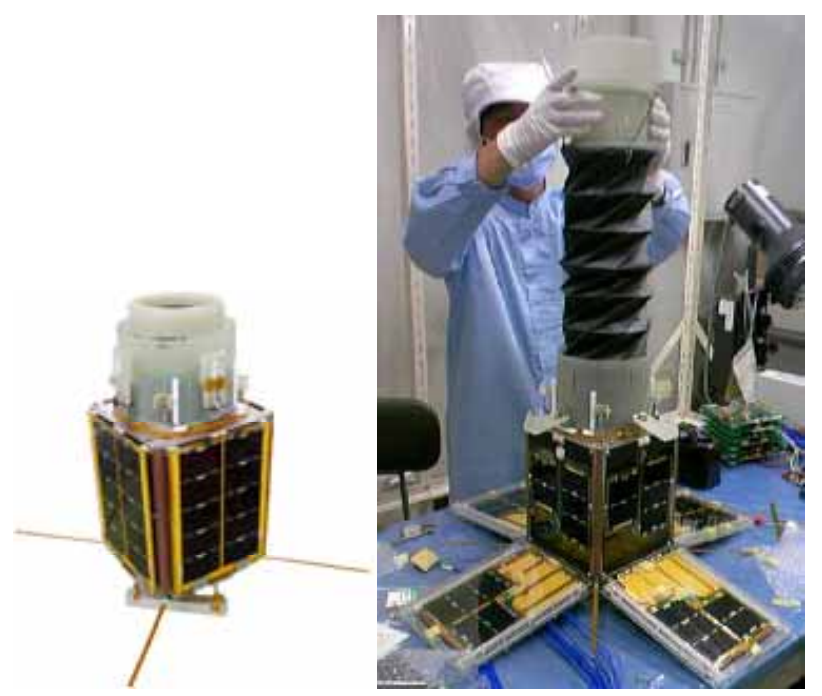

Fig. 1. PRISM (The boom is deployed in the right figure)

\section{Technological Challenges}

The key features of the extensible optical system are rigid disks, known as baffles, that are connected by a series of helical elastic glass-fiber rods and short cords. During the launch, the structural elements are compressed and the disks are sandwiched together. Upon reaching orbit, deployment occurs and the rods extend until their elongation is restricted by tension in the cords.

Based on this concept, we solved the three main technological challenges to achieve the requirements of the mission, which are related to precision, light-shielding and deployment. 


\subsection{Precision requirement of the structure}

A critical requirement of the structure is that it must keep its shape with a high degree of accuracy to satisfy the optics requirements. However, for simplicity of the system, no actuator can be used to control the shape of the boom. Therefore the boom's shape is directly defined by its mechanical characteristics, and the elastic forces of the glass-fiber rods and short cords.

To solve this problem, we simulated a variety of structures to find the optimal value of parameters such as contortion ratio, number of rods, rod length, number of cords, cord length, and the interval between each baffle. A key feature of the structure is that the main forces are limited to the axial direction of the extension. This makes it possible to compensate for fabrication errors and uncertainty related to the strong nonlinearity of the structural mechanics. Through iterations with the simulation, we selected the optimal parameters of the structural design. According to these parameters, we fabricated the structure of the boom whose static behavior was almost the same as predicted in the simulator.

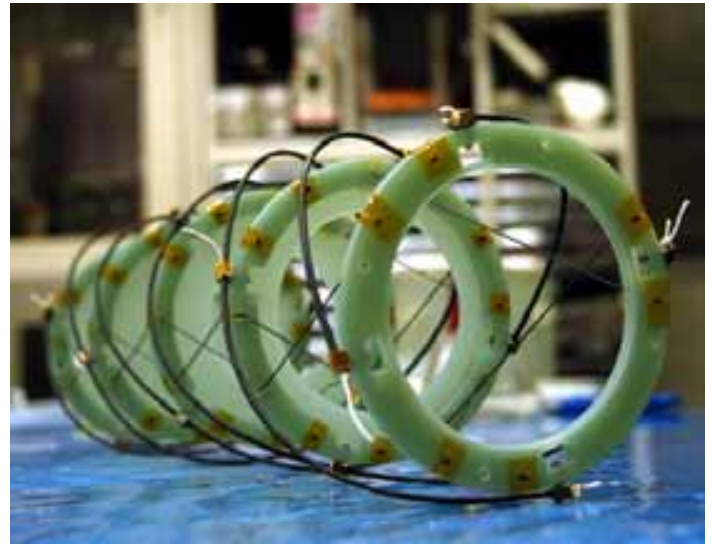

Fig. 2. Flame structure of the boom

\subsection{Achievement of light shielding by Origami}

For precise image acquisition, the boom should be shielded from stray light. Generally, the source of stray light coming is the sun and the earth's albedo. Any light reaching the image sensor that did not pass though the lens aperture will deteriorate the image. Therefore, the structure needs a protective cover satisfying the conditions below:

- as compactly and foldable as the frame structure,

- must not constrain the deployment of the main structure,

- inner surface must be non-reflective,

- material has low outgas in orbit,

- survivable in space environment (radiation, ultra-violet ray etc.).

The light shielding material also plays an important role in reducing thermal deformation of the boom's structure.

As mentioned above, the boom is deployed by unscrewing itself, implying that the shielding cover must also have a degree of rotational freedom. The boom and its cover should also have a matching ratio of contortion per deployed length.
To achieve this, we introduce an idea inspired by "Origami”, a traditional paper art in Japan. Origami techniques are proficient at folding thin materials which can be applied to the idea of a foldable cylinder.
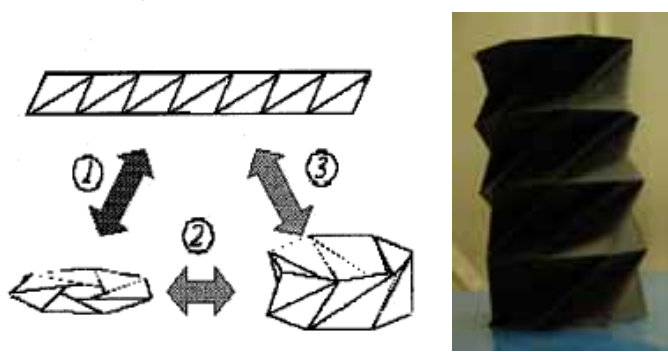

Fig. 3. Folding procedure of cylinder (Hagiwara et el, 2004) and light shield fabricated for the flame structure

As shown in Fig. 3, the key feature of foldable cylinder is that its deployment can be achieved by unscrewing itself following the motion of the boom inside. Also the glass-fiber rods of boom's structure can be stored in the folded valleys of the cylinder. This leads to a compact and effective cover design. Corresponding to the parameters of the boom obtained in 2.1, we designed the parameters of the foldable cylinder, including the rhombus shape and number between baffle intervals. Subject to the conditions above, we chose a thin, mat processed, and low outgas film for the material of the shield. The product is made by black pet film, which satisfies the important conditions of the optical system.

\subsection{Development of housing mechanism}

The contracted boom must be stored in a compact way and released smoothly. The impact of the deployment must also be adequately absorbed. Also considering the ease of assembly, we developed a housing mechanism as shown in Fig. 4. Inside the housing, the boom is compactly folded and grasped on its lens hood by four craws. The craws are latched by a fishing line, which will be cut by nichrome wire once in orbit to release the boom. To design the shape of craw, we referred the latch of marman band in which as the fishing line is cinched tight, the hood is strongly fixed to it. The housing mechanism is attached to the satellite only by four springs. It enables an easy attachment and makes it possible to use the same system for other remote sensing satellites. The spring is also intended to absorb the impact of deployment.

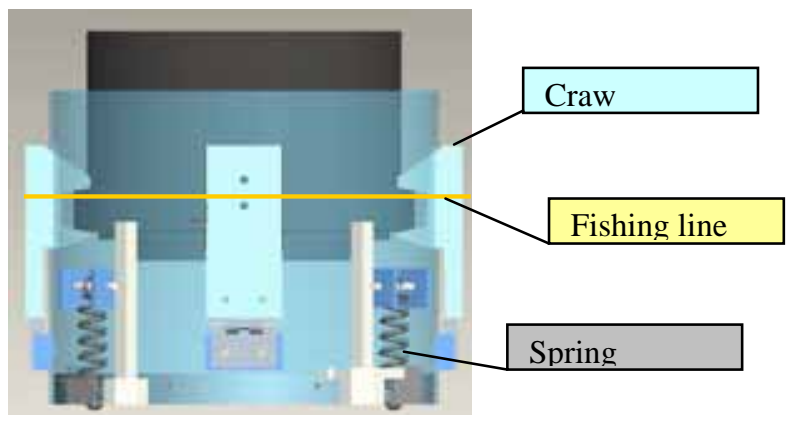

Fig. 4. Housing Mechanism 


\section{Micro Gravity Experiment}

\subsection{Experimental objectives}

In September 2007, we conducted micro-gravity experiments at Diamond Air Service in Nagoya, Japan using a Gulf Stream-II jet tuned for parabolic flights. The experiment was promoted by the Japan Space Forum. During the parabolic flights, we tested the boom deployment under micro-gravity conditions.

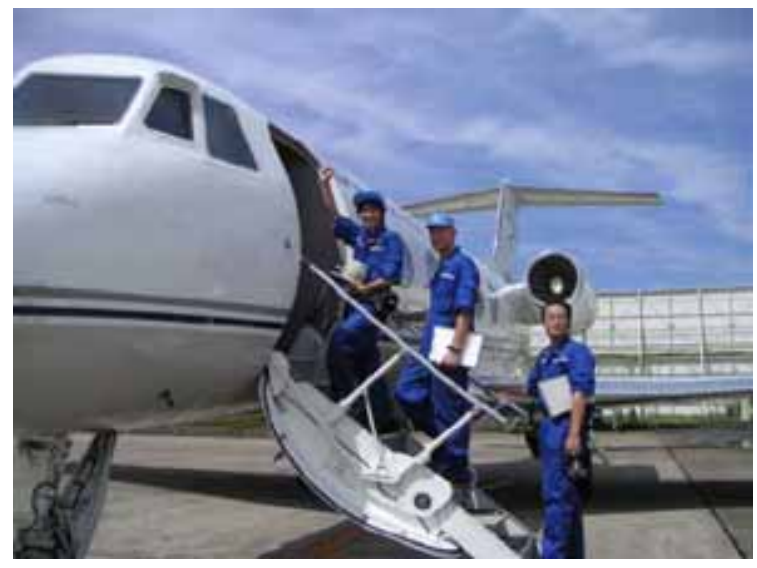

Fig. 5. Gulf Stream-II and crews

The objectives of the experiments were as follows:

- to determine if the boom has enough internal force to deploy without being helped by gravity,

- to estimate the difference in boom shape after deployment in gravity and in-orbit,

- to observe the repeatability of the shape following successive deployments,

- to observe vibration and damping of the boom immediately after the deployment,

- to analyze the vibration characteristics of the deployed boom in micro-gravity ${ }^{7}$.

The boom was mechanically and geometrically optimized as an optical system, however, it is difficult to determine the in-orbit behavior of the total structure because of its flexibility and strong nonlinearity. Therefore, operational and system identification tests under micro gravity are needed.

\subsection{Experimental method}

The engineering model of the boom, whose design is almost the same as that of the flight model, was used for the experiments. In the aircraft, two test racks were used to test the boom structure. This paper describes the experiments taken in the first rack while in the other rack, vibration tests were conducted. ${ }^{7}$.

\subsubsection{Deployment experiment}

Among a total of 38 parabolic cycles onboard four different flights, we conducted the deployment experiment 26 times. Fig. 6 shows the schematics of the experiment. The housing mechanism storing the stowed boom is attached to the upper plate with a spring as it would be attached to the surface of satellite. The upper plate is connected to the ceiling of the rack structure with spacers on which strain gauges are attached to measure the impact caused by deployment.

Each parabolic sequence created approximately 15 seconds of micro-gravity conditions. After 5 seconds of micro-gravity, the boom extension is initiated by cutting the fishing line. The motion of deployment is captured by three cameras. Three laser sensors and one video camera are attached at the bottom of the rack. Laser sensors measure the distance to the tip of the boom, while the video camera captures the spotted light of the sensors on the boom tip where tick marks on two directions are printed. By using the calibrated sensors and the video images, length and tilting of the boom can be estimated. The two other video cameras are attached around the exterior of the boom. One is to record the behavior of the housing mechanism while the other is for observing the motion of the boom from a diagonal perspective. The acceleration of the aircraft is also measured along its pitch and roll axes.

\subsubsection{Observation experiment}

In the remaining 12 micro-gravity sequences, the behavior of the elongated boom in response to the vibration caused by the aircraft disturbances was observed. The sensors used in the experiment are the same as those used in the deployment experiment.

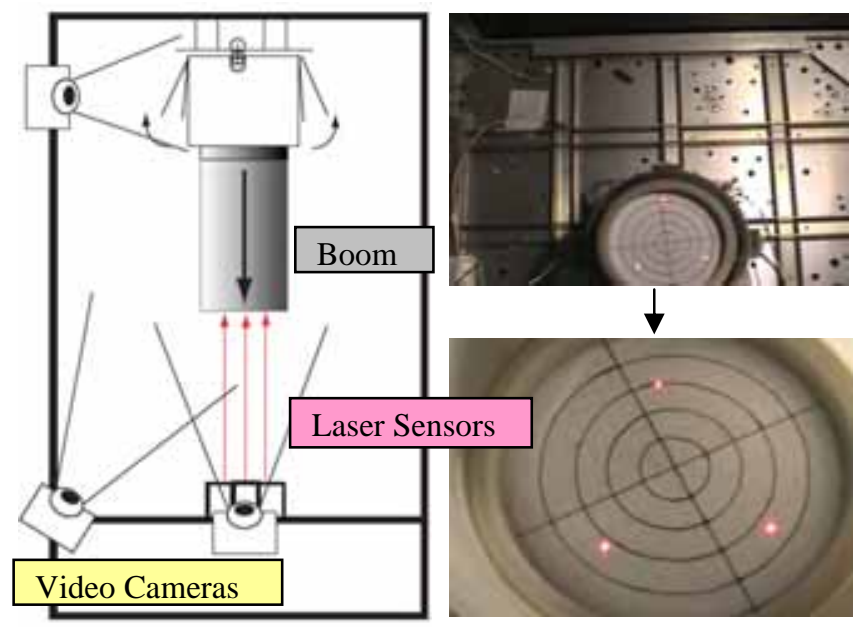

Fig. 6. Experimental schematics

(Red points are spotted light from laser sensors)

\subsection{Experimental result}

\subsubsection{Overall behavior of the deployment}

In 26 tests, the deployment under micro-gravity was successful all but two times in which the boom didn't deploy because of an operator mistake. Fig. 7 shows a sequence of a deployment under micro-gravity. The behavior of the boom includes the characteristics below: 


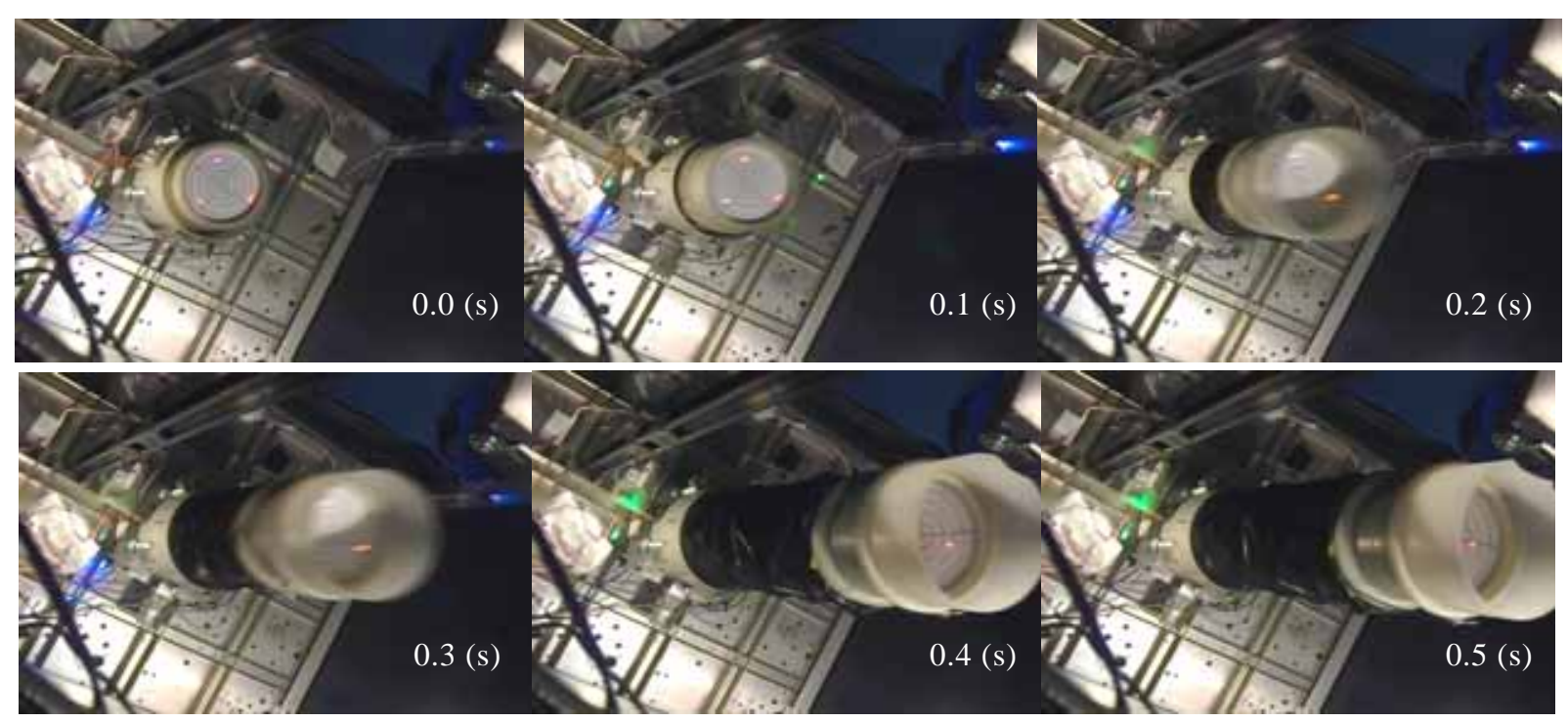

Fig. 7. Deployment of the boom under micro gravity

- Deployment speed was slower than expected. It took about 0.5 second to deploy.

- Boom exhibited prolonged vibration after deployment.

- When the vibration eventually stopped, bending of the boom was observed.

Fig. 8 and Fig. 9 show the boom length measured by the three sensors. Fig. 8 shows a stable deployment, and Fig. 9 shows an undamped case where excessive vibration disrupted the laser sensors. We suggest the following two reasons for this behavior:

- The impact of deployment caused the vibration which wasn't damped by the end of stable micro gravity.

- Micro-gravity experiment inside aircraft had non-negligible acceleration disturbances.

In order to see whether the vibration was caused by the impact of deployment or by the aircraft disturbance, we decided to conduct the observation experiment in 12 flights.

\subsubsection{Effects of aircraft disturbance}

To consider the vibration and bending caused after the deployment, the effects of aircraft disturbance (remnant acceleration) are analyzed. From results of the flight tests, it was observed that aircraft disturbance caused measurable boom motion. When the remnant accelerations were small, the boom's motion stopped immediately after the deployment, and in the observation experiments, the boom remained still. This implies that the boom has enough damping to respond to the deployment impact. Fig.10 shows a comparison of the aircraft disturbance in two axes of a stable case and an undamped case. As shown in the graphs, the frequency of vibration in the direction of the roll axis (x-axis) is higher than that in the direction of the pitch axis (y-axis), and closer to the natural frequency (about $1.7 \mathrm{~Hz})^{7)}$ of the boom.

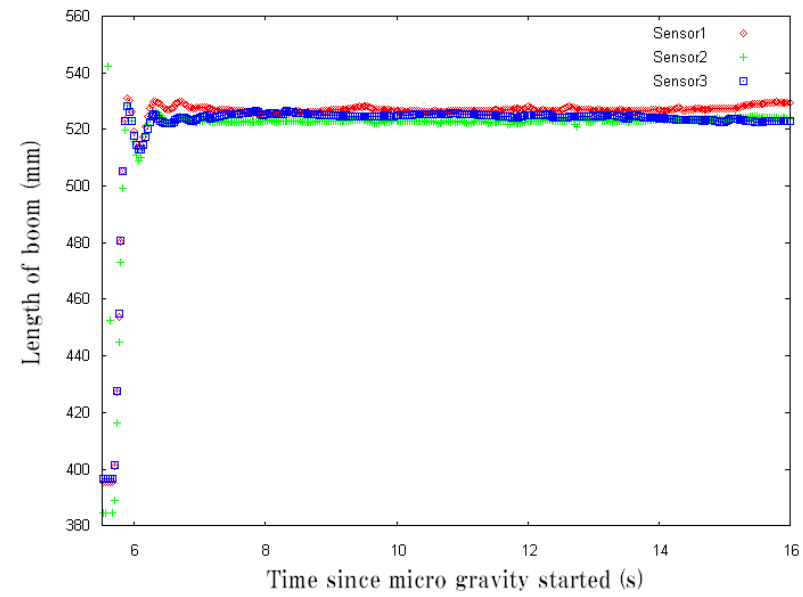

Fig.8. Boom length measured by laser Sensors (Stable case)

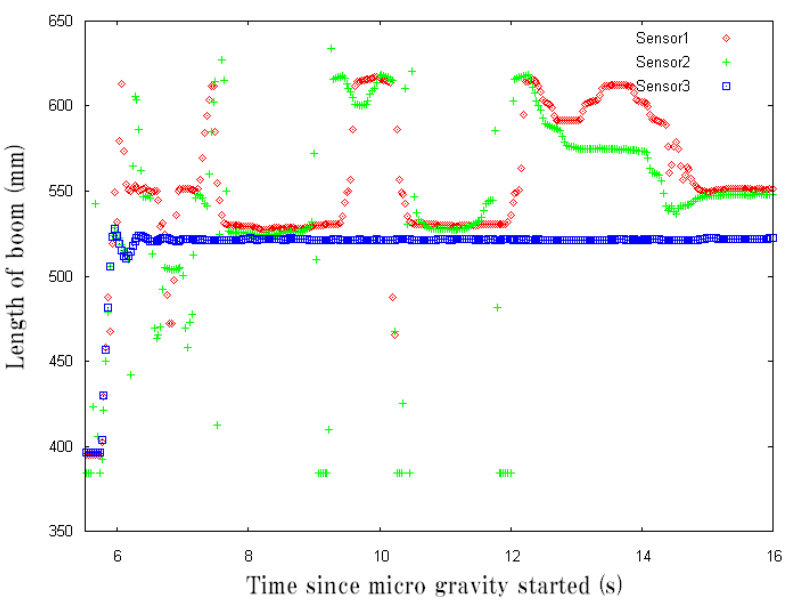

Fig. 9. Boom length measured by laser sensors (Undamped case) 


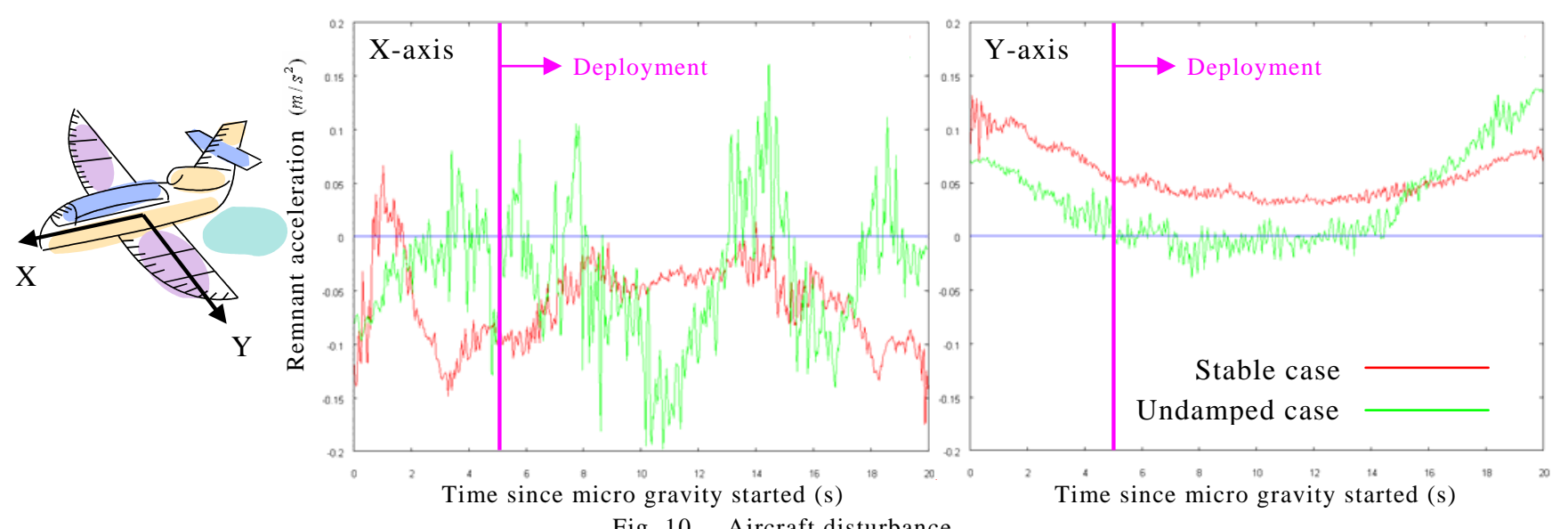

This suggests that the vibration of boom is mainly caused by the aircraft disturbance in the $\mathrm{x}$-axis as the disturbance in the y-axis did not show much effect. Another characteristic of the disturbance is that it has a bias component which would cause the bending of the boom.

\subsubsection{Estimation of the deformation in orbit}

As we discussed in the previous section, the boom has enough damping to respond to the deployment impact, but it is sensitive to disturbances. To estimate the resulting vibration and bending in orbit, we consider the magnitude of torque acting on the tip of boom and the magnitude of deformation in the experiment. The torque is calculated from the approximated magnitude of disturbance and approximated boom length. The magnitude of deformation is approximated from the motion taken by video camera. Their values are shown in Table 1.

Table 1. Torque and deformation in the experiment

\begin{tabular}{|c|c|}
\hline $\begin{array}{l}\text { Torque caused by aircraft } \\
\text { disturbance }\end{array}$ & $\begin{array}{l}\text { Magnitude of undesirable } \\
\text { deformation }\end{array}$ \\
\hline $0.1(\mathrm{~N}) \times 0.5(\mathrm{~m}) \approx 10^{-2}(\mathrm{Nm})$ & $10^{-2}(\mathrm{~m})$ \\
\hline
\end{tabular}

The torque acting on the tip of boom in orbit can be estimated from the magnetic disturbance and attitude control of the satellite (both of which are very small).

Table 2. The order of magnitude in orbit

\begin{tabular}{|c|c|}
\hline $\begin{array}{l}\text { Torque caused by magnetic } \\
\text { disturbance }\end{array}$ & $\begin{array}{l}\text { Torque caused by attitude } \\
\text { control }\end{array}$ \\
\hline $10^{-6}(\mathrm{Nm})$ & $10^{-5}(\mathrm{Nm})$ \\
\hline
\end{tabular}

If the relationship between the magnitude of torque and the magnitude of deformation is linear and also if the motion of the boom in aircraft was not affected by air drug, the deformation in orbit should be negligible.

\subsubsection{Length of the boom}

Before the micro gravity experiment was conducted, it was expected that the boom would come to rest immediately after the deployment, enabling us to measure its static shape with the three laser sensors and video camera. But as mentioned above, it was almost impossible to measure the boom shape under the sufficiently damped condition.

For this reason, we consider that the boom shape becomes almost straight when the length measured by three laser sensors is the closest, and thus define the "boom length" as the average of three lengths measured in the straight condition. Because of this, tilting of the boom tip in a static state is not obtained.

Boom length was calculated for each parabolic cycle, concluding that the average boom length in the observation experiments is longer than in the deployment experiments by about $2 \mathrm{~mm}$. The results are shown in Table 3 and Table 4 .

Table 3. Boom length obtained from the deployment experiments

\begin{tabular}{|l|l|}
\hline Average & $524.94 \mathrm{~mm}$ \\
\hline Standard deviation & $0.44 \mathrm{~mm}$ \\
\hline
\end{tabular}

Note) Deployment experiment means that the boom is deployed from sowed position during micro-gravity period.

Table 4. Boom length obtained from the observation experiments

\begin{tabular}{|l|l|}
\hline Average & $526.86 \mathrm{~mm}$ \\
\hline Standard deviation & $0.64 \mathrm{~mm}$ \\
\hline
\end{tabular}

Note) Observation experiment means that the boom is initially extended and its vibration is observed during micro-gravity period.

This implies that the structure of the light shield is constraining the boom length after deployment. In gravity, the boom is tensioned by the lens weight to be straight as the short cords are tensioned well. However in the dynamic deployment under micro-gravity, the extending force is not as strong and as a result the extension is constrained somewhat by the light shield. This suggests that during the deployment experiments, the boom length was measured while the boom had not been fully extended.

On the other hand, in the observation experiments, the deployed boom is tensioned during a $2 \mathrm{G}$ state immediately 
before the micro-gravity starts, so the boom is more than fully extended and the shield constraint is of no consequence.

Apart from the results obtained from the deployment experiments, we use the boom length obtained from the observation experiments to estimate the boom length in orbit. Because the constraint is not a factor, the boom length is purely decided by the force balance between the glass-fiber rods and short cords, which should be replicated in the flight model.

Considering the boom length measured in gravity, we conclude that the boom length in orbit is shorter than the boom length in gravity by $3.0 \mathrm{~mm}$. To compensate for the 3.0 $\mathrm{mm}$ of offset, a spacer has been inserted to the lens case of the flight model.

Table 5. Results of boom length measurement

\begin{tabular}{|l|l|}
\hline \multicolumn{2}{|c|}{ Boom length } \\
\hline Average Length in $\mathrm{G}$ & $529.86 \mathrm{~mm}$ \\
\hline Average Length in $\mu \mathrm{G}$ & $526.86 \mathrm{~mm}$ \\
\hline Offset & $-3.0 \mathrm{~mm}$ \\
\hline Standard deviation in $\mu \mathrm{G}$ & $0.75 \mathrm{~mm}$ \\
\hline
\end{tabular}

In the flight model, fabrication of the light shield was improved to reduce the constraining force, however it may still be present. It is thought that boom length in orbit would be between that obtained in the deployment experiment and that obtained in the observation experiments. To compensate for this constraint and for possible discrepancies of the calculated boom length, the satellite has focusing mechanism to shift the position of image sensor by $+-3.0 \mathrm{~mm}$ in the axial direction.

\section{Conclusion and Future Work}

From the design for base of the boom structure in the breadboard model, we developed the engineering model of the extendable boom to satisfy the requirements for a space telescope. However, it is difficult to uniquely determine the in-orbit behavior of the boom structure because of its complexity, flexibility and strong nonlinearity. To confirm our new design by more than calculations and simulations, micro-gravity experiments were conducted where we deployed the boom in micro-gravity. Several important results were obtained related to the axial contraction of the boom, the constraint imposed by the light shield, and the behavior of the boom's transverse vibration.

Based on the test results, we have developed the flight model of the boom. It is now attached to the satellite waiting for the impending launch.

Other unknown variables of the boom motion exist, including the tilting of the tip of the boom or the thermal deformation. At this stage, we can only predict the behavior of these variables with a simulation or other experiments using the engineering model.

After the launch, all predicted parameters should be compared with the data collected from the real system. The method for determining the important parameters from the telemetry will be included in our future works. For instance, the position of the focusing mechanism while taking an in-focus photograph will provide information about the boom's parameters. Also, three wide angle cameras are attached around the boom to help observe the motion of the boom on orbit.

The results of the comparison should be fed back to the process of the development, design and experimental analysis. We hope that this technological demonstration will provide effective design criteria for similar remote sensing satellites which use deployable structures.

\section{Acknowledgement}

This research was supported by "Ground-based Research Program for Space Utilization” promoted by Japan Space Forum.

\section{References}

1) Segert, T. et al : Dobson Space Telescope -Recent Developments, The 56th International Astronautical Congress, Fukuoka, Japan, IAC-05-B5.4.08(2005)

2) Tsuda, Y. et al : University of Tokyo's CubeSat Project -Its Educationnal and Technological significance -, The $15^{\text {th }}$ Annual AIAA/USU Conference on Small Satellites, SSC01-Viiib-7(2001).

3) Funase, R. et al : Technology demonstration on University of Tokyo's pico-satellite "XI-V" and its effective operation result using ground station network, Acta Astronautica, 61(2007), pp. 707-711.

4) Komatsu, M.: University of Tokyo Nano Satellite Project "PRISM", The $26^{\text {th }}$ International Symposium on space Technology ans Science, 2008-s-08.

5) Nakamura, Y. et al : Extensible Boom-Based Optical System for Nano-Scale Remote Sensing Satellite "PRISM", 19th Annual AIAA/USU Conference on Small Satellite, SSC05-VI-1(2005)

6) Hagiwara, I. et al : Optimization for Crush Characteristics of Cylindrical Structure Using Reversed Spiral Model, Transaction of the Japan Society of Mechanical Engineers. A, 70(2004), pp.36-42

7) Lambert, C. et al : Vibration Analysis of Nanosatellite PRISM's Extendable Boom, The $26^{\text {th }}$ International Symposium on space Technology and Science, 2008-m-13. 